\title{
Phase Retrieval with Random Phase Illumination
}

\author{
Albert Fannjiang ${ }^{1, *}$ and Wenjing Liao ${ }^{1}$ \\ 1 Department of Mathematics, University of California, Davis, CA 95616 \\ *Corresponding author: fannjiang@math.ucdavis.edu
}

\begin{abstract}
This paper presents a detailed, numerical study on the performance of the standard phasing algorithms with random phase illumination (RPI). Phasing with high resolution RPI and the oversampling ratio $\sigma=4$ determines a unique phasing solution up to a global phase factor. Under this condition, the standard phasing algorithms converge rapidly to the true solution without stagnation. Excellent approximation is achieved after a small number of iterations, not just with high resolution but also low resolution RPI in the presence of additive as well multiplicative noises. It is shown that RPI with $\sigma=2$ is sufficient for phasing complex-valued images under a sector condition and $\sigma=1$ for phasing nonnegative images. The Error Reduction algorithm with RPI is proved to converge to the true solution under proper conditions.

(c) 2018 Optical Society of America
\end{abstract}

OCIS codes: $100.5070,170.1630,340.7430$.

\section{Introduction}

Fourier phase retrieval is the problem of reconstructing an unknown image from its Fourier magnitude data. Phase retrieval is fundamental in many applications such as X-ray crystallography [2], astronomy [3], coherent light microscopy [4], quantum state tomography and remote sensing. 
Due to the absence of the phase information, phase retrieval does not have a unique solution. Phase retrieval literature has long settled with the notion of uniqueness modulo the trivial ambiguities of spatial shift, conjugate inversion and global phase [5] [6] and focused on circumventing the stagnation problem associated with the standard phasing algorithms. The numerical stagnation problem is often attributed to the nonconvex constraint imposed by the Fourier magnitude data [7] [8] [9] [10].

In this paper, we explore a phasing method based on random phase modulator which randomly modifies the phases of the original image by a mask. As proved in [1] phasing with random (phase or amplitude) illumination often leads to a unique solution up to a global phase factor (here dubbed absolute uniqueness). In what follows we show that phasing with random phase illumination (RPI) also leads to superior numerical performances, including rapid convergence, much reduced data and noise stability of the standard algorithms. We show that under proper conditions the Error-Reduction (ER) algorithm with RPI converges to the true solution (Theorem 4).

Consider the discrete version of the phase retrieval problem: Let $\mathbf{n}=\left(n_{1}, \ldots, n_{d}\right) \in \mathbb{Z}^{d}$ and $\mathbf{z}=\left(z_{1}, \ldots, z_{d}\right) \in \mathbb{C}^{d}$. Define the multi-index notation $\mathbf{z}^{\mathbf{n}}=z_{1}^{n_{1}} z_{2}^{n_{2}} \ldots z_{d}^{n_{d}}$. Let $\mathcal{C}(\mathcal{N})$ denote the set of finite complex-valued functions on $\mathbb{Z}^{d}$ vanishing outside

$$
\mathcal{N}=\{\mathbf{0} \leq \mathbf{n} \leq \mathbf{N}\}, \quad \mathbf{N}=\left(N_{1}, N_{2}, \ldots, N_{d}\right)
$$

Here $\mathbf{m} \leq \mathbf{n}$ if $m_{j} \leq n_{j}, \forall j$. Denote $|\mathcal{N}|=\prod_{j=1}^{d} N_{j}$.

The $z$-transform of a $d$ dimensional finite array $f(\mathbf{n}) \in \mathcal{C}(\mathcal{N})$ is given by

$$
F(\mathbf{z})=\sum_{\mathbf{n}} f(\mathbf{n}) \mathbf{z}^{-\mathbf{n}}
$$

The Fourier transform can be obtained from the $z$-transform as

$$
F\left(e^{i 2 \pi \boldsymbol{\omega}}\right)=\sum_{\mathbf{n}} f(\mathbf{n}) e^{-2 \pi i \boldsymbol{\omega} \cdot \mathbf{n}}
$$

for $\boldsymbol{\omega}=\left(\omega_{1}, \omega_{2}, \ldots, \omega_{d}\right), 0 \leq \omega_{j}<1$.

From the calculation

$$
\left|F\left(e^{i 2 \pi \mathbf{w}}\right)\right|^{2}=\sum_{\mathbf{n}=-\mathbf{N}} \sum_{\mathbf{m}+\mathbf{n} \in \mathcal{N}} f(\mathbf{m}+\mathbf{n}) \overline{f(\mathbf{m})} e^{-\mathrm{i} 2 \pi \mathbf{n} \cdot \mathbf{w}}
$$

we see that the Fourier magnitude measurement is equivalent to the standard discrete Fourier measurement of the correlation function

$$
\mathcal{C}_{f}(\mathbf{n})=\sum_{\mathbf{m} \in \mathcal{N}} f(\mathbf{m}+\mathbf{n}) \overline{f(\mathbf{m})}
$$


if sampled at the lattice

$$
\mathcal{L}=\left\{\boldsymbol{\omega}=\left(\omega_{1}, \ldots, \omega_{d}\right) \mid \omega_{j}=0, \frac{1}{2 N_{j}+1}, \frac{2}{2 N_{j}+1}, \ldots, \frac{2 N_{j}}{2 N_{j}+1}\right\}
$$

which is $2^{d}$ times of the grid of the original image. The standard phasing problem is to recover the array $f(\mathbf{n})$ from its Fourier intensity measurement $Y(\boldsymbol{\omega})=\left|F\left(e^{i 2 \pi \boldsymbol{\omega}}\right)\right|$ for $\boldsymbol{\omega} \in \mathcal{L}$ or smaller sampling sets.

Clearly the correlation function $\mathcal{C}_{f}$ and the Fourier magnitude data are invariant under spatial translation

$$
f(\cdot) \rightarrow f(\cdot+\mathbf{t}) \text { for some } \mathbf{t} \in \mathbb{Z}^{d},
$$

conjugate inversion

$$
f(\cdot) \rightarrow \overline{f(\mathbf{N}-\cdot)}
$$

and constant global phase change

$$
f(\cdot) \rightarrow e^{i \theta} f(\cdot)
$$

These trivial associates all share the same global geometric information as the original object. The classical results of uniqueness given in [5] 6] [12] say that for almost all objects in dimension two or higher the trivial associates are the only ambiguities there are with phase retrieval.

On the other hand, by dimension counting Miao et al. [11] have argued that overall 2 times oversampling, independent of the dimension $d$, uniquely determines a unique phasing solution up to spatial shift, conjugate inversion and global phase factor. To measure the degree of oversampling we use the oversampling ratio (OR)

$$
\sigma=\frac{\text { Fourier magnitude data number }}{\text { unknown-valued image pixel number }}
$$

introduced in [11]. As we demonstrate below, Miao et al.'s conjecture can be realized by using RPI, but not uniform illumination.

As shown in [1] random illumination (RI) can help remove the phasing ambiguities of spatial shift and conjugate inversion. An illumination amounts to replacing the original image $f(\mathbf{n})$ by

$$
g(\mathbf{n})=\lambda(\mathbf{n}) f(\mathbf{n}),
$$

where $\lambda(\mathbf{n})$ is a known array representing the incident wave. In the case of uniform illumination, $\lambda(\mathbf{n})=1$. In the case of random phase illumination (RPI) [13],

$$
\lambda(\mathbf{n})=e^{i \phi(\mathbf{n})}
$$


where $\phi(\mathbf{n})$ are random variables on $[0,2 \pi]$, and in the case of random amplitude illumination [14, 15], $\lambda(\mathbf{n})$ is an array of real random variables. RI can be facilitated by random phase/amplitude modulators or random masks.

The paper is organized as follows. We review the absolute uniqueness of phasing with RPI in Section 2 and standard phasing algorithms in Section 3 where convergence of the Error Reduction (ER) iteration to the true solution is presented (Theorem 4). We present the numerical phasing results in Section 4. We conclude in Section 5.

For the rest of the paper we use the following notation: the vector space $\mathcal{C}(\mathcal{N})$ is endowed with the inner product $\langle f, g\rangle=\sum_{\mathbf{n}} \overline{f(\mathbf{n})} g(\mathbf{n})$. For a complex number $z, \Re(z)$ and $\Im(z)$ denote the real and imaginary part of $z . \measuredangle z \in[0,2 \pi)$ denotes the phase (angle) of $z$. When $z=0, \measuredangle z$ is taken to be 0 unless specified otherwise. $[\alpha]=\alpha(\bmod (2 \pi))$.

\section{Uniqueness}

In the following we recall several uniqueness results from [1] relevant to phasing with RPI.

First we define the rank of an array. The support of the array consists of the set of nonzero pixels. The rank of the array is the dimension of its support's convex hull in $\mathbb{R}^{d}$.

Theorem 1. Let $\lambda(\mathbf{n})$ be independent, continuous random variables on $\mathbb{S}^{1}$. Let $f(\mathbf{n}) \in \mathcal{C}(\mathcal{N})$ be a real-valued array of rank $\geq 2$. Then, with probability one, $f$ is determined absolutely uniquely up to \pm sign by the Fourier magnitude measurement on $\mathcal{L}$.

A more general, practical constraint is to restrict the image values within a certain sector of the complex plane. For instance, when the incident X-rays are low energy photons(soft $\mathrm{X}$-rays), the electron density is complex. The real part represents the effective number of electrons that diffract the X-rays in phase and is usually positive but becomes negative only when the energy of the incident $\mathrm{X}$-rays is near an absorption edge. The imaginary part represents the absorption of the X-rays by the specimen and thus is always positive.

Theorem 2. Let $\lambda(\mathbf{n})$ be independent, continuous random variables on $\mathbb{S}^{1}$. Let $f$ be a complex-valued array of rank $\geq 2$ such that $\measuredangle f(\mathbf{n}) \in[\alpha, \beta], \forall \mathbf{n}$. Let $S$ denote the sparsity of the image and let $\lfloor S / 2 \rrbracket$ be the greatest integer less than or equal to $S / 2$.

Suppose that the phases $\phi(\mathbf{n})$ of RPI are independent, uniform random variables on $[0,2 \pi]$. Then with probability no less than $1-|\mathcal{N}|(\beta-\alpha)^{\llbracket S / 2 \Downarrow}(2 \pi)^{-\llbracket S / 2 \Downarrow}$, the object $f$ is uniquely determined, up to a global phase, by the Fourier magnitude measurement on $\mathcal{L}$.

The global phase is uniquely determined if the angular sector $[\alpha, \beta]$ is tight in the sense that no proper subset of $[a, b]$ contains all the phases of the object. 
For general complex-valued images without any constraint, we use two independent RPIs to collect data.

Theorem 3. Let $\lambda_{1}(\mathbf{n})$ and $\lambda_{2}(\mathbf{n})$ be two independent arrays of continuous random variables on $\mathbb{S}^{1}$. Let $f(\mathbf{n}) \in \mathcal{C}(\mathcal{N})$ be any complex-valued array of rank $\geq 2$. Then almost surely $f(\mathbf{n})$ is uniquely determined, up to a constant phase factor, by the Fourier magnitude measurement on $\mathcal{L}$ with two illuminations $\lambda_{1}$ and $\lambda_{2}$. If the second illumination $\lambda_{2}(\mathbf{n})$ is deterministic while $\lambda_{1}(\mathbf{n})$ is random as above, then the same conclusion holds.

\section{Phasing Algorithms}

To find the true object satisfying both the object-domain constraint, which is usually convex, and the frequency-domain constraint, which is non-convex, most phasing algorithms are based on the idea of alternating projections from the convexity literature [9].

\section{A. Projections}

Defintion 1. Let $\mathcal{D}$ be a subset of $\mathcal{C}(\mathcal{N})$, the orthogonal projection of $f \in \mathcal{C}(\mathcal{N})$ on $\mathcal{D}$ is $\operatorname{argmin}_{g \in \mathcal{D}}\|g-f\|$

If the minimizer is not unique, one of them is arbitrarily selected. When $\mathcal{D}$ is a closed convex subset of $\mathcal{C}(\mathcal{N})$, the minimizer is unique.

Proposition 1. Let $\mathcal{D}$ denote any closed convex subset of $\mathcal{C}(\mathcal{N})$ and let $f$ be any element in $\mathcal{C}(\mathcal{N})$. Then there exits a unique $h \in \mathcal{D}$ such that

$$
i n f_{g \in \mathcal{D}}\|g-f\|=\|h-f\|
$$

Let $\Gamma$ be the set of functions satisfying the object-domain constraint, such as a known support or positivity, and $\Omega$ be the set of functions satisfying the frequency-domain constraint imposed by the known Fourier magnitude data. A solution of phase retrieval is a function belonging to $\Gamma \cap \Omega$. Let $\mathcal{P}_{o}$ and $\mathcal{P}_{f}$ be the orthogonal projection on $\Gamma$ and $\Omega$ respectively.

Let $\Lambda$ be the diagonal matrix with diagonal elements $\lambda(\mathbf{n})$, and set $g=\Lambda f$. Let $\Phi$ be the discrete Fourier transform and set $Y=|\Phi f|$.

Given the Fourier intensity data $Y$, we define the intensity fitting operator $\mathcal{T}$ as

$$
G^{\prime}(\boldsymbol{\omega})=\mathcal{T}\{G\}(\boldsymbol{\omega})= \begin{cases}Y(\boldsymbol{\omega}) e^{i \measuredangle G(\boldsymbol{\omega})} & \text { if }|G(\boldsymbol{\omega})|>0 \\ Y(\boldsymbol{\omega}) & \text { if }|G(\boldsymbol{\omega})|=0\end{cases}
$$


When $G(\boldsymbol{\omega})=0, \measuredangle G(\boldsymbol{\omega})$ is not uniquely defined and $\measuredangle G(\boldsymbol{\omega})$ is set 0 in (5). In this case,

$$
\mathcal{P}_{f}=\Lambda^{-1} \Phi^{-1} \mathcal{T} \Phi \Lambda
$$

Indeed $\measuredangle G(\boldsymbol{\omega})$ can be arbitrarily chosen at the zero set of $G$, and we define

$$
\mathcal{P}_{f}^{\theta}=\Lambda^{-1} \Phi^{-1} \mathcal{T}^{\theta} \Phi \Lambda
$$

where

$$
\mathcal{T}^{\theta}\{G\}(\boldsymbol{\omega})= \begin{cases}Y(\boldsymbol{\omega}) e^{i \measuredangle G(\boldsymbol{\omega})} & \text { if }|G(\boldsymbol{\omega})|>0 \\ Y(\boldsymbol{\omega}) e^{i \theta(\boldsymbol{\omega})} & \text { if }|G(\boldsymbol{\omega})|=0\end{cases}
$$

The object domain projection $\mathcal{P}_{o}$ can take a varied form depending on the problem.

- When $\Gamma$ is the set of images with a given phase $\alpha$,

$$
\mathcal{P}_{o}\{h\}(\mathbf{n})=\mathcal{P}_{\alpha}\{h(\mathbf{n})\}=\max \{\Im(h(\mathbf{n})) \sin \alpha+\Re(h(\mathbf{n})) \cos \alpha, 0\} e^{i \alpha} .
$$

- When $\Gamma$ is the set of images with phases in $[\alpha, \beta]$ for $0 \leq \alpha<\beta \leq 2 \pi$,

$$
\begin{aligned}
& - \text { if } \beta-\alpha \leq \pi, \mathcal{P}_{o}\{h\}(\mathbf{n})=\left\{\begin{array}{ll}
h(\mathbf{n}) & \text { if } \alpha \prec \measuredangle h(\mathbf{n}) \prec \beta \\
\mathcal{P}_{\beta}\{h(\mathbf{n})\} & \text { if } \beta \prec \measuredangle h(\mathbf{n}) \prec[\beta+\pi / 2] \\
\Re\left(\mathcal{P}_{\alpha}\{h(\mathbf{n})\}\right) & \text { if }[\alpha-\pi / 2] \prec \measuredangle h(\mathbf{n}) \prec \beta \\
0 & \text { else }
\end{array},\right. \\
& - \text { if } \beta-\alpha>\pi, \mathcal{P}_{o}\{h\}(\mathbf{n})=\left\{\begin{array}{ll}
h(\mathbf{n}) & \text { if } \alpha \prec \measuredangle h(\mathbf{n}) \prec \beta \\
\mathcal{P}_{\beta}\{h(\mathbf{n})\} & \text { if } \beta \prec \measuredangle h(\mathbf{n}) \prec[(\alpha+\beta) / 2+\pi] \\
\mathcal{P}_{\alpha}\{h(\mathbf{n})\} & \text { if }[(\alpha+\beta) / 2+\pi] \prec \measuredangle h(\mathbf{n}) \prec \alpha
\end{array},\right.
\end{aligned}
$$

where $a \prec \theta \prec b$ means $\theta$ is between $a$ and $b$ such that

$$
\left\{\begin{array}{ll}
a \leq \theta \leq b & \text { if } a \leq b \\
a \leq \theta<2 \pi \quad \text { or } 0 \leq \theta \leq b & \text { if } a>b
\end{array} .\right.
$$

- When $\Gamma$ is the set of real valued images,

$$
\mathcal{P}_{o}\{h\}(\mathbf{n})=\Re(h(\mathbf{n})) .
$$

- When $\Gamma$ is the set of nonnegative real-valued images,

$$
\mathcal{P}_{o}\{h\}(\mathbf{n})=\max \{\Re(h(\mathbf{n})), 0\} .
$$


- When $\Gamma$ is the set of complex valued images with nonnegative real and imaginary parts,

$$
\begin{aligned}
& \Re\left(\mathcal{P}_{o}\{h\}(\mathbf{n})\right)=\max (\Re(h(\mathbf{n})), 0) \\
& \Im\left(\mathcal{P}_{o}\{h\}(\mathbf{n})\right)=\max (\Im(h(\mathbf{n})), 0) .
\end{aligned}
$$

- When $\Gamma$ is the set of images with support $S$,

$$
\mathcal{P}_{o}\{h\}(\mathbf{n})=\left\{\begin{array}{ll}
h(\mathbf{n}) & \text { if } \mathbf{n} \in S \\
0 & \text { else }
\end{array} .\right.
$$

Two error metrics $\varepsilon_{o}$ and $\varepsilon_{f}$ defined by

$$
\begin{aligned}
& \varepsilon_{o}(h)=\left\|\mathcal{P}_{o}\{h\}-h\right\|, \\
& \varepsilon_{f}(h)=\left\|\mathcal{P}_{f}\{h\}-h\right\|
\end{aligned}
$$

play an important role of our studies. When $\Phi \Lambda$ is unitary, as in the case of RPI,

$$
\varepsilon_{f}(h)=\left\|\mathcal{P}_{f}\{h\}-h\right\|=\|\mathcal{T} \Phi \Lambda h-\Phi \Lambda h\|=\|Y-|\Phi \Lambda h|\| .
$$

\section{B. Oversampling}

The oversampling method has proven to be an effective, flexible way of implementing various phasing algorithms by converting Fourier magnitude data more finely sampled than demanded by the original image grid into zero padding which then acts like a support constraint of the original image [5, 16, 20, 21]. In this set-up, the oversampling ratio is given by

$$
\sigma=\frac{\text { image pixel number }+ \text { zero-padding pixel number }}{\text { image pixel number }} .
$$

\section{C. Error reduction (ER)}

ER algorithm [17] is based on the Gerchberg-Saxton algorithm [18] and is the most basic phasing algorithm. ER is the plain version of the alternated projection method:

$$
f_{k+1}=\mathcal{P}_{o} \mathcal{P}_{f} f_{k}
$$

which can be conveniently represented by the following diagram

ER enjoys the error-decreasing property following the same argument in [17]. 


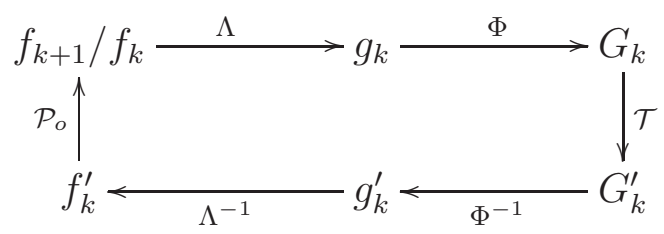

Fig. 1.

Proposition 2. Let $\Gamma$ be a closed convex subset of $\mathcal{C}(\mathcal{N})$. Let $\Phi$ and $\Lambda$ be unitary matrices. Then the array $\left\{f_{k}\right\}$ produced in (8) satisfies

$$
\varepsilon_{f}\left(f_{k+1}\right) \leq \varepsilon_{f}\left(f_{k}\right) .
$$

The equality holds if and only if $f_{k+1}=f_{k}$.

Proof.

$$
\begin{aligned}
\varepsilon_{f}\left(f_{k}\right) & =\left\|f_{k}-f_{k}^{\prime}\right\| \\
& \geq\left\|f_{k+1}-f_{k}^{\prime}\right\| \\
& =\left\|G_{k+1}-G_{k}^{\prime}\right\| \\
& \geq\left\|G_{k+1}-G_{k+1}^{\prime}\right\| \\
& =\left\|f_{k+1}-f_{k+1}^{\prime}\right\| \\
& =\varepsilon_{f}\left(f_{k+1}\right) .
\end{aligned}
$$

The equality holds only if $\left\|f_{k}-f_{k}^{\prime}\right\|=\left\|f_{k+1}-f_{k}^{\prime}\right\|$, where $f_{k+1}=\mathcal{P}_{o}\left\{f_{k}^{\prime}\right\}$. Since $\Gamma$ is a closed convex subset, $f_{k+1}=f_{k}$ according to Proposition 1.

Remark 1. Proposition $\mathbf{Q}$ holds for the $f_{k+1}=\mathcal{P}_{o} \mathcal{P}_{f}^{\theta} f_{k}$ with arbitrary $\theta(\boldsymbol{\omega})$.

Proposition 2 shows that the error $\varepsilon_{f}\left(f_{k}\right)$ decreases strictly until it reaches a fixed point of $\mathcal{P}_{o} \mathcal{P}_{f}$, implying that the ER iteration converges to a fixed point.

Proposition 3. Let $f_{k+1}=\mathcal{P}_{o} \mathcal{P}_{f} f_{k}$. Let $\Gamma$ be a closed convex subset of $\mathcal{C}(\mathcal{N})$ and $\Phi, \Lambda$ be unitary matrices. Then every convergent subsequence of $\left\{f_{k}\right\}$ converges to some $h$ such that

1. if $\Phi \Lambda h(\boldsymbol{\omega}) \neq 0, \forall \boldsymbol{\omega} \in \mathcal{L}, h$ is a fixed point of $\mathcal{P}_{o} \mathcal{P}_{f}$. 
2. if $\Phi \Lambda h(\boldsymbol{\omega})=0$ for some $\boldsymbol{\omega} \in \mathcal{L}, h$ is a fixed point of $\mathcal{P}_{o} \mathcal{P}_{f}^{\theta}$ for some $\theta$.

The proof of Proposition 3 is given in the Appendix. The question is, Is a fixed point of ER necessarily a phasing solution? With the uniform illumination, however, this is generally not true [19]. When a fixed point fails to be a phasing solution, it is called a trap and can plague the reconstruction procedure (cf. Figure 3(a), 4(a) and 4(c)).

Below, we answer this question in the affirmative under certain assumptions for the case of RPI. The difficulty is ER may converge to a fixed point of $\mathcal{P}_{o} \mathcal{P}_{f}^{\theta}$ which fails to satisfy the Fourier magnitude data. In other words, the limiting point $h$ may not be a fixed point of $\mathcal{P}_{f}^{\theta}$.

In the following main theoretical result of the paper, we prove that if $\mathcal{P}_{f}^{\theta} h$ satisfies the zero-padding condition, then it must be the phasing solution.

Theorem 4. Let $f(\mathbf{n}) \in \mathcal{C}(\mathcal{N})$ be an array with $f(\mathbf{0}) \neq 0$ and of rank $\geq 2$. Let $\lambda(\mathbf{n})$ be i.i.d. continuous random variables on $\mathbb{S}^{1}$. Let the Fourier magnitude be sampled on $\mathcal{L}$. Let $h$ be a fixed point of $\mathcal{P}_{o} \mathcal{P}_{f}^{\theta}$ such that $\mathcal{P}_{f}^{\theta} h$ satisfies the zero-padding condition.

(a) If $f$ is real-valued, $h= \pm f$ with probability one,

(b) If $f$ satisfies the sector condition of Theorem Q2, then $h=e^{i \nu} f$, for some $\nu$, and satisfies the same sector constraint with probability at least $1-|\mathcal{N}|(\beta-\alpha)^{\lfloor S / 2 \Downarrow}(2 \pi)^{-\lfloor S / 2 \Downarrow}$.

\section{D. $\mathrm{HIO}$}

The hybrid input-output (HIO) algorithm is a widely used, better-performing phasing method than ER's [17]. HIO differs from ER in how to update the image in the object domain in order to avoid the trapping and stagnation.

Below we present a modified version of Fienup's HIO which performs better than the original version. We refer to Figure 1 for the notation. In HIO, the last step $\mathcal{P}_{o}$ of ER iteration is replaced by the following.

- When $\Gamma$ is the set of real-valued images,

$$
\begin{aligned}
& \Re\left(f_{k+1}(\mathbf{n})\right)=\Re\left(f_{k}^{\prime}(\mathbf{n})\right) \\
& \Im\left(f_{k+1}(\mathbf{n})\right)=\Im\left(f_{k}(\mathbf{n})\right)-\beta \cdot \Im\left(f_{k}^{\prime}(\mathbf{n})\right),
\end{aligned}
$$

If, in addition, the nonnegativity constraint is assumed, then

$$
\begin{aligned}
& \Re\left(f_{k+1}(\mathbf{n})\right)=\left\{\begin{array}{ll}
\Re\left(f_{k}^{\prime}(\mathbf{n})\right) & \text { if } \Re\left(f_{k}^{\prime}(\mathbf{n})\right) \geq 0 \\
\Re\left(f_{k}(\mathbf{n})\right)-\beta \cdot \Re\left(f_{k}^{\prime}(\mathbf{n})\right) & \text { if } \Re\left(f_{k}^{\prime}(\mathbf{n})\right)<0
\end{array},\right. \\
& \Im\left(f_{k+1}(\mathbf{n})\right)=\Im\left(f_{k}(\mathbf{n})\right)-\beta \cdot \Im\left(f_{k}^{\prime}(\mathbf{n})\right) .
\end{aligned}
$$


- When $\Gamma$ is the set of complex-valued images with nonnegative real and imaginary parts,

$$
\begin{aligned}
& \Re\left(f_{k+1}(\mathbf{n})\right)=\left\{\begin{array}{ll}
\Re\left(f_{k}^{\prime}(\mathbf{n})\right) & \text { if } \Re\left(f_{k}^{\prime}(\mathbf{n})\right) \geq 0 \\
\Re\left(f_{k}(\mathbf{n})\right)-\beta \cdot \Re\left(f_{k}^{\prime}(\mathbf{n})\right) & \text { if } \Re\left(f_{k}^{\prime}(\mathbf{n})\right)<0
\end{array} .\right. \\
& \Im\left(f_{k+1}(\mathbf{n})\right)=\left\{\begin{array}{ll}
\Im\left(f_{k}^{\prime}(\mathbf{n})\right) & \text { if } \Im\left(f_{k}^{\prime}(\mathbf{n})\right) \geq 0 \\
\Im\left(f_{k}(\mathbf{n})\right)-\beta \cdot \Im\left(f_{k}^{\prime}(\mathbf{n})\right) & \text { if } \Im\left(f_{k}^{\prime}(\mathbf{n})\right)<0
\end{array} .\right.
\end{aligned}
$$

\section{E. Algorithms with two illuminations}

Let $\lambda_{1}(\mathbf{n})$ and $\lambda_{2}(\mathbf{n})$ be two arrays representing two illuminating fields. Two sets of Fourier magnitude data $Y_{1}=\left|\Phi \Lambda_{1} f\right|$ and $Y_{1}=\left|\Phi \Lambda_{2} f\right|$ are collected, each with an OR $\sigma$. Let $\mathcal{T}_{1}$ and $\mathcal{T}_{2}$ be the intensity fitting operators corresponding to $Y_{1}$ and $Y_{2}$, respectively, as in (5). Thus the projections onto the set of images satisfying the Fourier magnitude data $Y_{1}$ and $Y_{2}$ are, respectively,

$$
\mathcal{P}_{1}=\Lambda_{1}^{-1} \Phi^{-1} \mathcal{T}_{1} \Phi \Lambda_{1}
$$

and

$$
\mathcal{P}_{2}=\Lambda_{2}^{-1} \Phi^{-1} \mathcal{T}_{2} \Phi \Lambda_{2} .
$$

The corresponding ER algorithm with two sets of Fourier magnitude data $Y_{1}$ and $Y_{2}$ is given by

$$
f_{k+1}=\mathcal{P}_{o} \mathcal{P}_{2} \mathcal{P}_{1} f_{k} .
$$

The corresponding HIO is obtained by replacing $\mathcal{P}_{o}$ in (16) by (10)-(15).

\section{Numerical Simulations}

In this section, we perform numerical phasing from the Fourier intensity measurement with UI or RPI.

Our test images are the $256 \times 256$ Cameraman and the $138 \times 184$ Phantom. We surround both images by dark (i.e. zero-valued) border to create images of loose support. Images of loose support are typically more challenging to reconstruct. For Cameraman the border is 13 pixel wide in each dimension and the resulting image has $269 \times 269$ pixels in total. For Phantom the dark margin is such that the resulting image has $200 \times 200$ pixels.

For the oversampling ratio $\sigma$, we zero pad the images to generate a $269 \sqrt{\sigma} \times 269 \sqrt{\sigma}$ Cameraman and $200 \sqrt{\sigma} \times 200 \sqrt{\sigma}$ Phantom. We synthesize the Fourier magnitude data by applying the FFT to the array. 


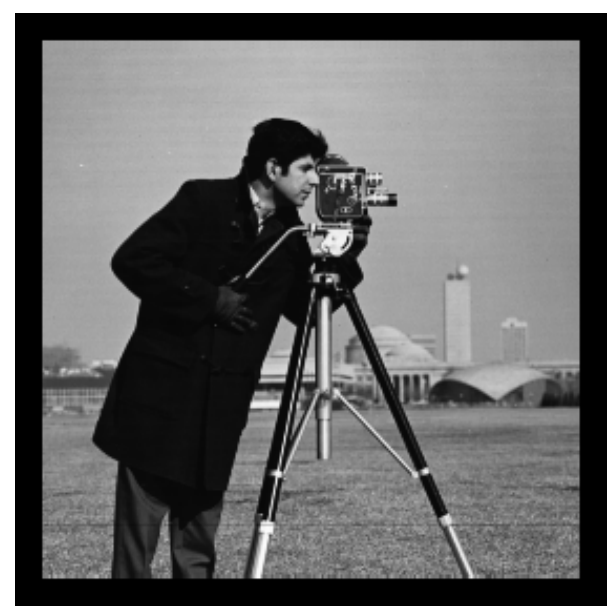

(a)

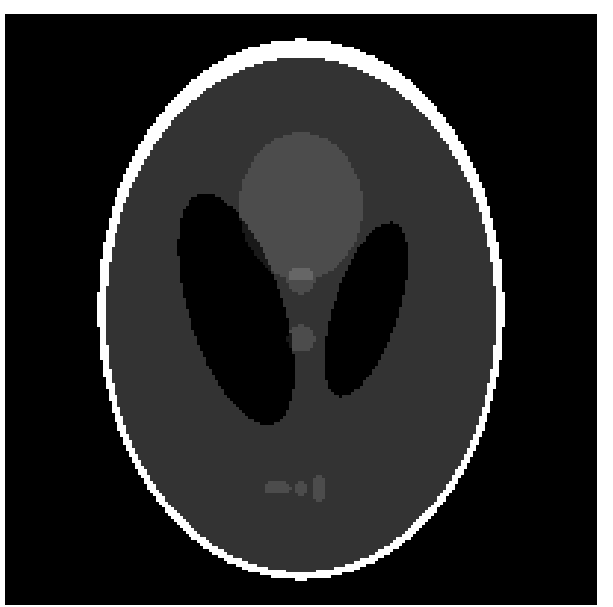

(b)

Fig. 2. Test images of loose support: (a) $269 \times 269$ Cameraman (b) $200 \times 200$ Phantom where the dark borders represent loose support.

\section{A. Error, Residual and Noise}

Let $\hat{f}$ be the recovered image. The relative error is defined as

$$
e(\hat{f})= \begin{cases}\|f-\hat{f}\| /\|f\| & \text { if absolute uniqueness holds } \\ \min _{\nu \in[0,2 \pi)}\left\|f-e^{i \nu} \hat{f}\right\| /\|f\| & \text { if uniqueness holds only up to a global phase }\end{cases}
$$

and the relative residual is defined as

$$
r(\hat{f})=\frac{\left\|Y-\left|\Phi \Lambda \mathcal{P}_{o}\{\hat{f}\}\right|\right\|}{\|Y\|}
$$

where $\mathcal{P}_{o}$ is introduced if $\hat{f}$ may not strictly satisfy the object domain constraint as in the case of HIO.

We consider three types of noise: Gaussian, Poisson and illumination noise, the last of which is defined as follows. Suppose the illumination field is noisy $\tilde{\lambda}(\mathbf{n})=\exp (i \tilde{\phi}(\mathbf{n}))$ with $\tilde{\phi}(\mathbf{n})=\phi(\mathbf{n})+t(\delta, \mathbf{n})$ where $t(\delta, \mathbf{n})$ are independent, uniform random variables in $[-\pi \delta / 100, \pi \delta / 100], \delta>0$.

We also test phasing with low resolution illumination which does not consist independently distributed pixel values but independently distributed blocks of deterministic (indeed, uniform) values. In our experiments, illumination of independent $40 \times 40$ blocks works well for 
real-valued nonnegative images and, for complex images, illumination of independent $4 \times 4$ blocks works well.

\section{B. Convergence Test}

The reconstruction of the real-valued nonnegative images Cameraman and Phantom with one UI or RPI is shown in Figures 3 and 4 respectively. For Figures 3 and 4 , we terminate the pure ER when $\left\|f_{k+1}-f_{k}\right\| /\left\|f_{k}\right\|<0.01 \%$. For HIO+ER, HIO is stopped when $\left\|f_{k+1}-f_{k}\right\| /\left\|f_{k}\right\|<$ $1 \%$ with a maximal 1000 iterations and ER is terminated when $\left\|f_{k+1}-f_{k}\right\| /\left\|f_{k}\right\|<0.01 \%$. In Figure 3 and 4 , $\left\|\hat{f}-\mathcal{P}_{o} \mathcal{P}_{f} \hat{f}\right\| /\|\hat{f}\|$ is as small as $0.01 \%$, implying that $\hat{f}$ is near a fixed point of $\mathcal{P}_{o} \mathcal{P}_{f}$.

As commented before the pure ER iteration always converges to a fixed point of $\mathcal{P}_{o} \mathcal{P}_{f}$. But with one uniform illumination and $\sigma=4$, the fixed point of $\mathcal{P}_{o} \mathcal{P}_{f}$ is not a phasing solution as the relative residual stagnates at 5.49\% in Figure 3(b) and at 14.71\% in Figure 4(b). HIO followed by ER improves the recovery over pure ER but the recovered Cameraman in Figure 3(c) displays the well known artifact of stripe pattern and the recovered Phantom in Figure 4(c) is severely blurred and distored.

With one low resolution RPI (block size: $40 \times 40$ ) and $\sigma=2$, the recovered images in Figure 3(e), 3(g), 4(e) and 4(g) are excellent approximation to the true images, even though absolute uniqueness is not guaranteed for low resolution RPI. HIO+ER is superior to pure ER in significant speed-up in convergence (Figure 3(f) versus 3(h), Figure 4(f) versus 4(h)).

With one high resolution RPI, high quality reconstruction can still be achieved with the oversampling ratio equal to 1, cf. Figures 3(i), 3(k), 4(i) and 4(k). Notice the rapid convergence of $\mathrm{HIO}+\mathrm{ER}$ in Figures 3 (l) and $4(1)$.

\section{C. Oversampling Ratio Test}

To systematically test the oversampling ratio required for phasing with RPI, we introduce $5 \%$ different types of noise (Gaussian, Poisson, Illumination), use low (block size: $40 \times 40$ ) as well as high resolution RPI and let $\sigma$ vary. We use an adaptive version of $\mathrm{HIO}+\mathrm{ER}$ : $\mathrm{HIO}$ and $\mathrm{ER}$ are terminated if the residual increases in 5 consecutive iterations. The relative error of reconstruction for the nonnegative image Phantom is averaged over 5 trials and shown in Figure 5(a). Clearly the relative error steadily decreases as the oversampling ratio increases. Without noise, low resolution RPI can achieve near zero error with $\sigma=1.1$. With $5 \%$ noise, the relative error stabilizes after $\sigma=2$ to a level comparable to the noise.

Next we consider the complex-valued Phantom with phases randomly distributed in the 
sector $[0, \pi / 2]$. Figure 5 (b) shows the average relative error $e(\hat{f})$ with one high resolution or low resolution (block size: $4 \times 4$ ) RPI and three kinds of noise. Again the relative error stabilizes after $\sigma=2$ to a level comparable to the noise. Note that for $1.8<\sigma<2$, there are more free variables in the complex-valued image than in the Fourier intensity data and yet the reconstructions are still of good quality.

Finally, we consider the complex-valued Phantom with phases random distributed in $[0,2 \pi]$. Figure $5(\mathrm{c})$ shows the average relative error $e(\hat{f})$ with one high resolution or low resolution (block size: $4 \times 4$ ) RPI plus one UI. Excellent recovery is achieved for $\sigma \geq 1.8$.

\section{D. Stability Test}

For images with positivity constraint and with one RPI, we terminate HIO when the relative residual increases for 5 consecutive steps and apply 10 steps of ER afterward. The maximal HIO iteration is set to be 100. For complex-valued images with two illuminations, we apply 200 steps of HIO and 300 steps of ER.

Figure 6 shows the recovery for the nonnegative-valued images with one high resolution RPI and 5\% Gaussian ((a)-(d)), Poisson ((e)-(h)) and illuminator noise ((i)-(l)). Multiplicative noise such as Poisson and illumination noises are generally more debilitating than the additive Gaussian noise.

With a low resolution RPI (block size: $40 \times 40$ ), the quality of reconstruction suffers slightly as shown in Figure 7 for nonnegative-valued images. The deterioration is most visible in the case of Poisson noise with the blocky pattern in Figure 7(e) and 7(g).

Figure 8 shows the average relative error $e(\hat{f})$ versus noise for (a) nonnegative-valued Phantom and $\sigma=2$, (b) Phantom with phases randomly distributed in $[0, \pi / 2]$ and $\sigma=4$ and (c) Phantom with phases randomly distributed in $[0,2 \pi]$ and $\sigma=3$. One high or low $(40 \times 40)$ resolution RPI is used in (a) while one high or low $(4 \times 4)$ RPI and one UI are used in (b) and (c). The adaptive HIO +50 ER is used for (a) and (b) while $200 \mathrm{HIO}+300$ ER is used for (c).

Relative error increases almost linearly with respect to the relative noise level with the noise amplification constant at worst 2. Clearly the illumination noise is most debilitating, followed by the Poisson noise. Nevertheless, the noise stability is achieved with even the low resolution RPI for all three types of noise. 


\section{Conclusion}

We have given a proof of convergence of ER (Theorem 4) and demonstrated that the stagnation problem of standard phasing algorithms such as ER and HIO can be alleviated if the ambiguities associated with spatial translation and conjugate inversion are removed by RPI. In addition, phasing with RPI has the following advantages: (i) It is stable with respect to additive as well as multiplicative noises with a moderate noise amplification constant; (ii) It reduces the oversampling ratio by more than a factor of 2; (iii) It reduces the number of iterations by more than an order of magnitude. We have also shown that phasing with RPI performs well with low resolution illumination and can tolerate a high level of illumination error, adding assurance that the random illumination needs not be calibrated exactly.

The lower bound $\sigma \geq 2$ for phasing of [11] was never actually achieved but we have achieved the lower limit in phasing with RPI for complex-valued images under a sector constraint. For nonnegative-valued images, phasing with one high resolution RPI reduces the oversampling ratio to unity, the minimum level by the dimensional count.

\section{Appendices}

\section{A. Proof of Proposition 3}

Proof. By Proposition 2, $\lim _{k \rightarrow \infty} \varepsilon_{f}\left(f_{k}\right)=\eta$, for some $\eta \geq 0$. Since $f_{k+1}=\mathcal{P}_{o}\left\{f_{k}^{\prime}\right\}$, we have $\left\|f_{k+1}\right\| \leq\left\|f_{k}^{\prime}\right\|=\left\|G_{k}^{\prime}\right\|=\|Y\|$ and that $\left\{f_{k}\right\}$ is a bounded sequence. Every bounded sequence in $\mathcal{C}(\mathcal{N})$ has a convergent subsequence, so $\left\{f_{k}\right\}$ has at least one convergent subsequence. Without loss of generality, we assume $\lim _{k \rightarrow \infty} f_{k}=f^{\star}$. Next, we prove that $f^{\star}$ must be a fixed point of $\mathcal{P}_{o} \mathcal{P}_{f}$ or $\mathcal{P}_{o} \mathcal{P}_{f}^{\theta}$ for some $\theta$.

Since $\Phi$ and $\Lambda$ are unitary matrices, $\lim _{k \rightarrow \infty} \Phi \Lambda f_{k}=\Phi \Lambda f^{\star}$ in $\|\cdot\|$, and thus

$$
\lim _{k \rightarrow \infty} \Phi \Lambda f_{k}(\boldsymbol{\omega})=\Phi \Lambda f^{\star}(\boldsymbol{\omega}), \forall \boldsymbol{\omega}
$$

- If $\Phi \Lambda f^{\star}(\boldsymbol{\omega})$ vanishes nowhere in $\mathcal{L}$, then

$$
\lim _{k \rightarrow \infty} \measuredangle \Phi \Lambda f_{k}=\measuredangle \Phi \Lambda f^{\star}
$$

implying

$$
\lim _{k \rightarrow \infty} G_{k}^{\prime}=\lim _{k \rightarrow \infty} \mathcal{T} \Phi \Lambda f_{k}=\mathcal{T} \Phi \Lambda f^{\star}
$$

Therefore,

$$
\lim _{k \rightarrow \infty} f_{k+1}=\mathcal{P}_{o} \mathcal{P}_{f} f^{\star}
$$


which along with the convergence of $\varepsilon_{f}\left(f_{k}\right)$ and $f_{k}$ implies that $\varepsilon_{f}\left(f^{\star}\right)=\varepsilon_{f}\left(\mathcal{P}_{o} \mathcal{P}_{f} f^{\star}\right)$. By Proposition 2, we have

$$
f^{\star}=\mathcal{P}_{o} \mathcal{P}_{f} f^{\star} .
$$

- If $\Phi \Lambda f^{\star}(\boldsymbol{\omega})=0$ at some $\boldsymbol{\omega} \in \mathcal{L}, \mathcal{T} \Phi \Lambda f_{k}(\boldsymbol{\omega})$ may not converge. However, since $\mathcal{T} \Phi \Lambda f_{k}$ is bounded in view of $\left\|\mathcal{T} \Phi \Lambda f_{k}\right\|=\|Y\|$, there exists a subsequence $\left\{f_{k_{j}}\right\}$ and some $\theta\left(f^{\star}\right)$ such that $\lim _{k \rightarrow \infty} \mathcal{T} \Phi \Lambda f_{k_{j}}(\boldsymbol{\omega})=\mathcal{T}^{\theta} \Phi \Lambda f^{\star}(\boldsymbol{\omega})$ where $\mathcal{T}^{\theta}$ is defined in (7). Therefore

$$
\lim _{j \rightarrow \infty} \mathcal{P}_{o} \Lambda^{-1} \Phi^{-1} \mathcal{T} \Phi \Lambda f_{k_{j}}=\mathcal{P}_{o} \Lambda^{-1} \Phi^{-1} \mathcal{T}^{\theta} \Phi \Lambda f^{\star},
$$

namely

$$
\lim _{j \rightarrow \infty} f_{k_{j}+1}=\lim _{j \rightarrow \infty} \mathcal{P}_{o} \mathcal{P}_{f} f_{k_{j}}=\mathcal{P}_{o} \mathcal{P}_{f}^{\theta} f^{\star}
$$

which along with the convergence of $\epsilon_{f}\left(f_{k}\right)$ and $f_{k}$ implies that $\varepsilon_{f}\left(f^{\star}\right)=\varepsilon_{f}\left(\mathcal{P}_{o} \mathcal{P}_{f}^{\theta} f^{\star}\right)$. By Proposition 2, it follows that

$$
f^{\star}=\mathcal{P}_{o} \mathcal{P}_{f}^{\theta} f^{\star} .
$$

\section{B. Proof of Theorem 4}

Define

$$
f_{\mathbf{m}+}(\cdot)=f(\mathbf{m}+\cdot), \quad f_{\mathbf{m}-}(\cdot)=f(\mathbf{m}-\cdot) .
$$

Let

$$
F(\mathbf{z})=\sum_{\mathbf{n}} f(\mathbf{n}) \mathbf{z}^{-\mathbf{n}}
$$

be the $z$-transform of $f$. According to the fundamental theorem of algebra, $F(\mathbf{z})$ can be written uniquely as

$$
F(\mathbf{z})=\alpha \mathbf{z}^{-\mathbf{n}_{0}} \prod_{k=1}^{p} F_{k}(\mathbf{z}),
$$

where $\mathbf{n}_{0}$ is a vector of nonnegative integers, $\alpha$ is a complex coefficient, and $F_{k}(\mathbf{z})$ are nontrivial irreducible monic polynomials in $\mathbf{z}^{-1}$.

Defintion 2 (Conjugate Symmetry). A polynomial $X(\mathbf{z})$ in $\mathbf{z}^{-1}$ is said to be conjugate symmetric if, for some vector $\mathbf{k}$ of positive integers and some $\theta \in[0,2 \pi)$,

$$
X(\mathbf{z})=e^{i \theta} \mathbf{z}^{-\mathbf{k}} \overline{X\left(\overline{\mathbf{z}}^{-1}\right)} .
$$


A conjugate symmetric polynomial may be reducible, irreducible, trivial, or nontrivial. If $A(\mathbf{z})$ is an arbitrary polynomial in $\mathbf{z}^{-1}$, then

$$
X(\mathbf{z})=A(\mathbf{z}) \cdot \mathbf{z}^{-\mathbf{N}} \overline{A\left(\overline{\mathbf{z}}^{-1}\right)}
$$

is conjugate symmetric. Any monomial $a \mathbf{z}^{\mathbf{k}}$ is conjugate symmetric.

The uniqueness of recovering a real-valued object from its Fourier magnitude or phase only data is discussed in [5] and can be easily generalized to the case of complex-valued objects.

Proposition 4. Let $f(\mathbf{n}) \in \mathcal{C}(\mathcal{N})$ be a finite array whose z-transform is irreducible up to a power of $\mathbf{z}^{-1}$. If the Fourier transform $G$ of $g(\mathbf{n}) \in \mathcal{C}(\mathcal{N})$ satisfies $\left|G\left(e^{i 2 p i \boldsymbol{\omega}}\right)\right|=$ $\left|F\left(e^{i 2 \pi \boldsymbol{\omega}}\right)\right|, \forall \boldsymbol{\omega} \in \mathcal{L}$, then $\exists \theta \in[0,2 \pi)$ and $\mathbf{m}$ such that either $g=e^{i \theta} f_{\mathbf{m}+}$ or $g=e^{i \theta} \overline{f_{\mathbf{m}-}}$.

Proposition 5. Let $f \in \mathcal{C}(\mathcal{N})$ be a finite array whose z-transform has no nontrivial conjugate symmetric factors. If $g \in \mathcal{C}(\mathcal{N})$ satisfies $\measuredangle F\left(e^{2 \pi i \boldsymbol{\omega}}\right)=\measuredangle G\left(e^{2 \pi i \boldsymbol{\omega}}\right), \forall \boldsymbol{\omega} \in \mathcal{L}$, then $g=\beta f$ for some real positive number $\beta$.

Proof. Consider the array $h$ defined by

$$
h(\mathbf{n})=f(\mathbf{n}) \star \overline{g(-\mathbf{n})}
$$

whose $z$-transform is

$$
H(\mathbf{z})=F(\mathbf{z}) \overline{G\left(\overline{\mathbf{z}}^{-1}\right)} .
$$

Since the phase of the Fourier transform of $h(\mathbf{n})$ is equal to

$$
\measuredangle H\left(e^{2 \pi i \boldsymbol{\omega}}\right)=\measuredangle F\left(e^{2 \pi i \boldsymbol{\omega}}\right)-\measuredangle G\left(e^{2 \pi i \boldsymbol{\omega}}\right),
$$

it follows that if $\measuredangle F\left(e^{2 \pi i \boldsymbol{\omega}}\right)=\measuredangle G\left(e^{2 \pi i \boldsymbol{\omega}}\right)$, then $\measuredangle H\left(e^{2 \pi i \boldsymbol{\omega}}\right)=0$. Thus the Fourier transform of $h$ is real-valued, implying that

$$
H(\mathbf{z})=\overline{H\left(\overline{\mathbf{z}}^{-1}\right)}
$$

Therefore,

$$
F(\mathbf{z}) \overline{G\left(\overline{\mathbf{z}}^{-1}\right)}=\overline{F\left(\overline{\mathbf{z}}^{-1}\right)} G(\mathbf{z}) .
$$

Multiplying both sides of (18) by $\mathbf{z}^{-\mathbf{N}}$ results in the following polynomial equation in $\mathbf{z}^{-1}$ :

$$
F(\mathbf{z}) \overline{G\left(\overline{\mathbf{z}}^{-1}\right)} \mathbf{z}^{-\mathbf{N}}=\overline{F\left(\overline{\mathbf{z}}^{-1}\right)} G(\mathbf{z}) \mathbf{z}^{-\mathbf{N}}
$$

Since $F(\mathbf{z})$ does not have trivial factors or nontrivial conjugate symmetric factors, we have

$$
F(\mathbf{z})=a \prod_{k} F_{k}(\mathbf{z})
$$


where $F_{k}(\mathbf{z})$ are nontrivial irreducible non-conjugate symmetric monic polynomials in $\mathbf{z}^{-1}$. Thus

$$
\mathbf{z}^{-\mathbf{N}} \overline{F\left(\overline{\mathbf{z}}^{-1}\right)}=a^{\prime} \mathbf{z}^{-m^{\prime}} \prod_{k} \tilde{F}_{k}(\mathbf{z})
$$

where $\tilde{F}_{k}(\mathbf{z})$ are the nontrivial irreducible non-conjugate symmetric monic polynomials in $\mathbf{z}^{-1}$ of the form $\tilde{F}_{k}(\mathbf{z})=\mathbf{z}^{-\mathbf{N}+\mathbf{p}_{k}} \overline{F_{k}\left(\overline{\mathbf{z}}^{-1}\right)}$ for some vector $\mathbf{p}_{k}$ of positive integers.

Writing

$$
G(\mathbf{z})=b \mathbf{z}^{-n} \prod_{\ell} G_{\ell}(\mathbf{z})
$$

where $G_{\ell}(\mathbf{z})$ are nontrivial irreducible monic polynomials in $\mathbf{z}^{-1}$, we have

$$
\mathbf{z}^{-\mathbf{N}} \overline{G\left(\overline{\mathbf{z}}^{-1}\right)}=b^{\prime} \mathbf{z}^{-n^{\prime}} \prod_{\ell} \tilde{G}_{\ell}(\mathbf{z})
$$

where $\tilde{G}_{\ell}(\mathbf{z})$ are the nontrivial irreducible monic polynomials in $\mathbf{z}^{-1}$ of the form $\tilde{G}_{\ell}(\mathbf{z})=$ $\mathbf{z}^{-\mathbf{N}+\mathbf{q}_{\ell}} \overline{G_{\ell}\left(\overline{\mathbf{z}}^{-1}\right)}$ for some vector $\mathbf{q}_{\ell}$ of positive integers.

Plugging (20), (21), (22) and (23) in (19) yields

$$
a b^{\prime} \mathbf{z}^{-n^{\prime}} \prod_{k} F_{k}(\mathbf{z}) \prod_{\ell} \tilde{G}_{\ell}(\mathbf{z})=a^{\prime} b \mathbf{z}^{-m^{\prime}-n} \prod_{k} \tilde{F}_{k}(\mathbf{z}) \prod_{\ell} G_{\ell}(\mathbf{z}) .
$$

Each nontrivial irreducible factor $F_{k}(\mathbf{z})$ must be equal to some $\tilde{F}_{k^{\prime}}(\mathbf{z})$ or some $G_{\ell^{\prime}}(\mathbf{z})$. However, if $F_{k}(\mathbf{z})=\tilde{F}_{k}(\mathbf{z})$, then $F_{k}(\mathbf{z})$ itself is conjugate symmetric. If, on the other hand, $F_{k}(\mathbf{z})=\tilde{F}_{k^{\prime}}(\mathbf{z})$ for some $k^{\prime} \neq k, F_{k}(\mathbf{z}) F_{k^{\prime}}(\mathbf{z})=\tilde{F}_{k^{\prime}}(\mathbf{z}) F_{k^{\prime}}(\mathbf{z})$ becomes a conjugate symmetric factor. Both cases, however, are excluded by the assumption that the $z$-transform of $f$ does not have conjugate symmetric factors. Thus each $F_{k}(\mathbf{z})$ must be equal to $G_{\ell^{\prime}}(\mathbf{z})$ for some $\ell^{\prime}$ and $F(\mathbf{z})$ so that $G(\mathbf{z})$ must be related by

$$
G(\mathbf{z})=Q(\mathbf{z}) \prod_{k} F_{k}(\mathbf{z})=\frac{1}{a} Q(\mathbf{z}) F(\mathbf{z}) .
$$

However, $G(\mathbf{z})$ and $F(\mathbf{z})$ are both polynomials in $\mathbf{z}^{-1}$, and since $F(\mathbf{z})$ contains no trivial factors, so $Q(\mathbf{z})$ must be a polynomial of $\mathbf{z}^{-1}$. Furthermore, plugging (25) in(18) yields

$$
Q(\mathbf{z})=\overline{Q\left(\overline{\mathbf{z}}^{-1}\right)}
$$

Therefore, $Q(\mathbf{z})=\beta$ and the theorem follows by noting that $\beta$ must be positive if $\measuredangle F(\boldsymbol{\omega})=$ $\measuredangle G(\omega)$. 
We next show that the $z$-transform of $\{\lambda(\mathbf{n}) f(\mathbf{n})\}$ is almost surely irreducible up to a power $\mathbf{z}^{-1}$ and not conjugate symmetric.

Lemma 1. Let $f \in \mathcal{C}(\mathcal{N})$ be a complex-valued array. Let $\{\lambda(\mathbf{n})\}$ be independent and continuous random variables on $\mathbb{S}^{1}$. Then, $\forall \mathbf{t} \neq \mathbf{0}$, the z-transform of $(\lambda f)_{\mathbf{t}+}$ and $\overline{(\lambda f)_{\mathbf{t}-}}$ is almost surely not conjugate symmetric.

Proof. Let

$$
\tilde{f}_{\mathbf{t}+}(\mathbf{n})=\lambda(\mathbf{t}+\mathbf{n}) f(\mathbf{t}+\mathbf{n})
$$

whose $z$-transform is

$$
\tilde{F}_{\mathbf{t}+}(\mathbf{z})=\sum_{\mathbf{n}} \lambda(\mathbf{t}+\mathbf{n}) f(\mathbf{t}+\mathbf{n}) \mathbf{z}^{-\mathbf{n}}
$$

$\tilde{F}_{\mathbf{t}+}(\mathbf{z})$ is conjugate symmetric if

$$
\tilde{F}_{\mathbf{t}+}(\mathbf{z})=e^{i \theta} \mathbf{z}^{-\mathbf{k}} \overline{\tilde{F}_{\mathbf{t}+}\left(\overline{\mathbf{z}}^{-1}\right)}
$$

for some vector $\mathbf{k}$ of positive integers and some $\theta \in[0,2 \pi)$. Plugging (26) in (27) yields

$$
\sum_{\mathbf{n}} \lambda(\mathbf{t}+\mathbf{n}) f(\mathbf{t}+\mathbf{n}) \mathbf{z}^{-\mathbf{n}}=e^{i \theta} \mathbf{z}^{-\mathbf{k}} \sum_{\mathbf{n}^{\prime}} \overline{\lambda\left(\mathbf{t}+\mathbf{n}^{\prime}\right) f\left(\mathbf{t}+\mathbf{n}^{\prime}\right)} \mathbf{z}^{\mathbf{n}^{\prime}}
$$

which implies

$$
\lambda(\mathbf{t}+\mathbf{n}) f(\mathbf{t}+\mathbf{n})=e^{i \theta} \overline{\lambda(\mathbf{t}+\mathbf{k}-\mathbf{n}) f(\mathbf{t}+\mathbf{k}-\mathbf{n})}, \forall \mathbf{n} .
$$

However, $f$ is deterministic, and $\lambda(\mathbf{n})$ are independent and continuous random variables, so (28) fails with probability one for any $\mathbf{k}$. There are finitely many choices of $\mathbf{k}$, so the $z$-transform of $(\lambda f)_{\mathbf{t}+}$ is almost surely not conjugate symmetric.

Similarly, the $z$-transform of $\overline{(\lambda f)_{\mathbf{t}-}}$ is also almost surely not conjugate symmetric.

Lemma 2. Let $f \in \mathcal{C}(\mathcal{N})$ be a complex-valued array of rank $\geq 2$. Let $\{\lambda(\mathbf{n})\}$ be independent and continuous random variables on $\mathbb{S}^{1}$. Then, the z-transform of $\{\lambda(\mathbf{n}) f(\mathbf{n})\}$ is irreducible up to a power of $\mathbf{z}^{-1}$ with probability one.

For the proof of Lemma 2 see Theorem 2 of [1].

Lemma 3. Let $f$ and $h$ be two complex-valued arrays. Let $\Phi$ be the discrete Fourier operator such that $\Phi f(\boldsymbol{\omega})=\sum_{\mathbf{k}} e^{-2 \pi i \boldsymbol{\omega} \cdot \mathbf{k}} f(\mathbf{k})$. Then $\measuredangle \Phi f_{\mathbf{t}+}=\measuredangle \Phi$ implies that $\measuredangle \Phi f=\measuredangle \Phi h_{(-\mathbf{t})+}$.

Proof. Note that

$$
\Phi f_{\mathbf{t}+}(\boldsymbol{\omega})=e^{2 \pi i \mathbf{t} \cdot \boldsymbol{\omega}} \Phi f(\boldsymbol{\omega})
$$


which implies

$$
2 \pi \mathbf{t} \cdot \boldsymbol{\omega}+\measuredangle \Phi f(\boldsymbol{\omega})(\bmod 2 \pi)=\measuredangle \Phi h(\boldsymbol{\omega})
$$

by the assumption $\measuredangle \Phi f_{\mathbf{t}+}=\measuredangle \Phi h$. Thus

$$
\measuredangle \Phi f(\boldsymbol{\omega})=\measuredangle \Phi h(\boldsymbol{\omega})-2 \pi \mathbf{t} \cdot \boldsymbol{\omega}(\bmod 2 \pi)
$$

which is equivalent to

$$
\measuredangle \Phi f=\measuredangle \Phi h_{(-\mathbf{t})+} \cdot
$$

Let us now turn to the proof of Theorem 4.

Proof. Let $f$ be the true image and $h$ a fixed point of the ER iteration. Suppose that $h^{\prime}=\mathcal{P}_{f}^{\theta} h$ satisfies the zero-padding condition. Then the following three equations hold:

$$
\begin{aligned}
\mathcal{P}_{o} h^{\prime} & =h \\
\left|\Phi \Lambda h^{\prime}\right| & =|\Phi \Lambda f| \\
\measuredangle \Phi \Lambda h^{\prime} & =\measuredangle \Phi \Lambda h
\end{aligned}
$$

According to Lemma 2, the $z$-transform of $\Lambda f$ is irreducible up to a power of $\mathbf{z}^{-1}$ with probability one, so there exists some integer-valued vector $\mathbf{m}$ with $-\mathbf{N} \leq \mathbf{m} \leq \mathbf{0}$ and some $\nu \in[0,2 \pi)$ such that

$$
h^{\prime}=e^{i \nu} \Lambda^{-1} \Lambda_{\mathbf{m}+} f_{\mathbf{m}+}
$$

or

$$
h^{\prime}=e^{i \nu} \Lambda^{-1} \overline{\Lambda_{\mathbf{m}-} f_{\mathbf{m}-}} .
$$

In the case of $h^{\prime}=e^{i \nu} \Lambda^{-1} \Lambda_{\mathbf{m}+} f_{\mathbf{m}+}$, the third equation in (31) becomes

$$
\measuredangle e^{i \nu} \Phi \Lambda_{\mathbf{m}+} f_{\mathbf{m}+}=\measuredangle \Phi \Lambda h .
$$

By Lemma 3 ,

$$
\measuredangle e^{i \nu} \Phi \Lambda f=\measuredangle \Phi \Lambda_{(-\mathbf{m})+} h_{(-\mathbf{m})+} \cdot
$$

Lemma 1 and 2, together with the assumption that $f(\mathbf{0}) \neq 0$ imply that the $\mathbf{z}$-transform of $\Lambda f$ is an irreducible, nontrivial and non-conjugate symmetric polynomial of $\mathbf{z}^{-1}$ with probability one.

Next, we apply Proposition 5 to (32). Both $\Lambda f$ and $\Lambda_{(-\mathbf{m})+} h_{(-\mathbf{m})+}$ are supported on a subset of $\{\mathbf{n} \mid-\mathbf{N} \leq \mathbf{n} \leq \mathbf{N}\}$. By Proposition 5, we obtain

$$
\gamma e^{i \nu} \Lambda f=\Lambda_{(-\mathbf{m})+} h_{(-\mathbf{m}-)+}
$$


or equivalently

$$
h(\mathbf{n})=\gamma e^{i \nu} \frac{\lambda(\mathbf{n}+\mathbf{m})}{\lambda(\mathbf{n})} f(\mathbf{n}+\mathbf{m})
$$

for some positive number $\beta$.

(a) If the true image $f(\cdot)$ is real-valued, then $h=\mathcal{P}_{o} h^{\prime}$ is real-valued, which by the proof of Theorem 1 (see Corrolary 1 of [1]) implies that $\nu=0, \pi$ and $\mathbf{m}=\mathbf{0}$ or equivalently

$$
h= \pm \gamma f
$$

with probability one. Plugging (33) in $\mathcal{P}_{o} \mathcal{P}_{f} h=h$ yields $\gamma=1$ and thus $h= \pm f$ with probability one.

(b) If $f$ satisfies the sector condition of Theorem 2, then $h=\mathcal{P}_{o} h^{\prime}$ satisfies the same sector condition which by the proof of Theorem 2 (see Theorem 4 (i) of [1]) implies that $\mathbf{m}=\mathbf{0}$ and

$$
h=\gamma e^{i \nu} f
$$

with probability at least $1-|\mathcal{N}|(\beta-\alpha)^{\lfloor S / 2 \Downarrow}(2 \pi)^{-\llbracket S / 2 \Downarrow}$. Plugging (34) in $\mathcal{P}_{o} \mathcal{P}_{f} h=h$ yields $\gamma=1$ and thus $h=e^{i \nu} f$.

By the similar argument one reaches the same conclusion in the case of $h^{\prime}=$ $e^{i \nu} \Lambda^{-1} \Lambda_{\mathbf{m}-} f_{\mathbf{m}-}$.

\section{References}

1. A. Fannjiang, "Absolute uniqueness of phase retrieval with random illumination," arXiv:1110.5097, to appear in Inverse Problems.

2. R. P. Millane, "Phase retrieval in crystallography and optics" J. Opt. Soc. Am.A vol. 7, pp. 394-411, 1990.

3. J. C. Dainty and J. R. Fienup, "Phase retrieval and image reconstruction for astronomy," Chapter 7 in H. Stark, ed., Image Recovery: Theory and Application vol. 3, pp. 231-275, Academic Press, 1987.

4. J. Miao, P. Charalambous, J. Kirz and D. Sayre, "Extending the methodology of X-ray crystallography to allow imaging of micrometre-sized non-crystalline specimens," Nature vol. 400, pp. 342-344, 1999.

5. M. Hayes, "The reconstruction of a multidimensional sequence from the phase or magnitude of its Fourier Transform," IEEE Trans. Acoust. Speech and Sign. Proc. vol. 30, pp. 140-154, 1982. 
6. M. Hayes, "Reducible Polynomials in More Than One Variables," Proc. IEEE vol. 30, pp. 197-198, 1982.

7. J. R. Fienup and C. C. Wackerman, "Phase-retrieval stagnation problems and solutions," J. Opt. Soc. Am. A vol. 3, pp. 1897-1907, 1986.

8. J. R. Fienup, "Reconstruction of a complex-valued object from the modulus of its Fourier transform using a support constraint," J. Opt. Soc. Am. A vol. 4, No. 1, pp. 118-123, 1987.

9. H. H. Bauschke, P. L. Combettes and D. R. Luke, "Phase retrieval, error reduction algorithm, and Fienup variants: a view from convex optimization," J. Opt. Soc. Am. A vol. 19, No. 7, pp. 13341-1345, 2002.

10. S. Marchesini, "A unified evaluation of iterative projection algorithms for phase retrieval," Rev. Sci. Instr. vol. 78, pp. 011301, 2007.

11. J. Miao, D. Sayre and H.N. Chapman, "Phase retrieval from the magnitude of the Fourier transforms of nonperiodic objects," J. Opt. Soc. Am. A 15 1662-1669 (1998).

12. T. A. Pitts and J. F. Greenleaf, "Fresnel transform phase retrieval from magnitude," IEEE Trans. Ultrasonics, Ferroelec. Freq. Contr. vol. 50, pp. 1035-1045, 2003.

13. P. F. Almoro, G. Pedrine, P. N. Gundu, W. Osten and S. G. Hansom, "Enhanced wavefront reconstruction by random phase modulation with a phasediffuser," Opt. Laser Eng. vol. 49, pp. 253-257, 2011.

14. A. Anand, G. Pedrini, W. Osten and P. Almoro, "Wavefront sensing with random amplitude mask and phase retrieval," Opt. Lett. vol. 32, pp. 1584-1586, 2007.

15. E. J. Candès, Y. Eldar, T. Strohmer and V. Voroninski, "Phase retrieval via matrix completion," preprint, August 2011.

16. J. Miao, and D. Sayre, "On possible extensions of X-ray crystallography through diffraction-pattern oversampling," Acta Cryst. A vol. 56, pp. 596-605, 2000.

17. J. R. Fienup, "Phase retrieval algorithms: a comparison," Appl. Opt. vol. 21, pp. 27582769, 1982.

18. R. W. Gerchberg and W. O. Saxton, "A practical algorithm for the determination of the phase from image and diffraction plane pictures," Optik 35, pp. 237, 1972.

19. A. Levi and H. Stark, "Image restoration by the method of generalized projections with application to restoration from magnitude," J. Opt. Soc. Am. 1(2), pp. 932-943,1984.

20. J. Miao, J. Kirz and D. Sayre, "The oversampling phasing method," Acta Cryst. D vol. 56, pp. 1312-1315, 2000.

21. J. Miao and D. Sayre and H. N. Chapman, "Phase retrieval from the magnitude of the Fourier transforms of nonperiodic objects," J. Opt. Soc. Am. A vol. 15, pp. 1662-1669, 
1998.

22. S. Marchesini, "Ab initio compressive phase retrieval," arXiv: 0809.2006v1, 2008.

23. Henry N. Chapman, A. Barty, S. Marchesini, A. Noy, S. P. Hau-Riege, C. Cui, M. R. Howells, R. Rosen, H. He, J. Spence, U. Weierstall, T. Beetz, C. Jacobsen and D. Shapiro, "High-resolution ab initio three-dimensional x-ray diffraction microscopy," J. Opt. Soc. Am. A vol. 23, No. 5, pp. 1179-1200, 2006. 


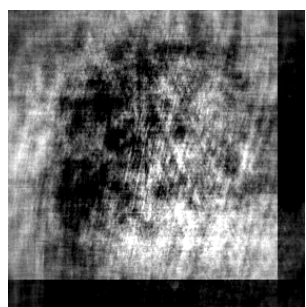

(a)

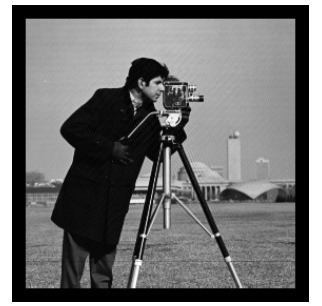

(e)

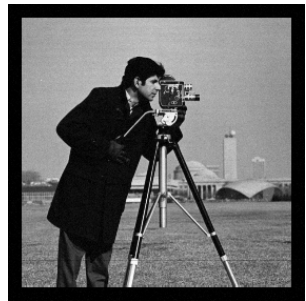

(i)

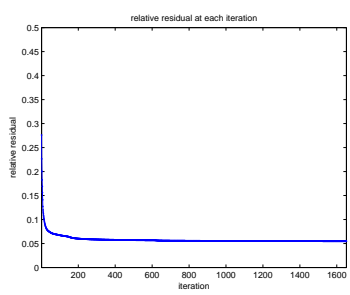

(b)

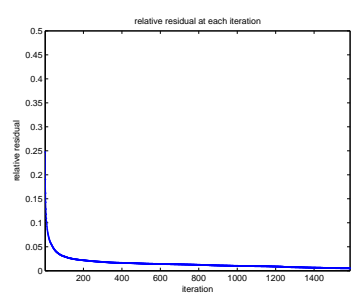

(f)

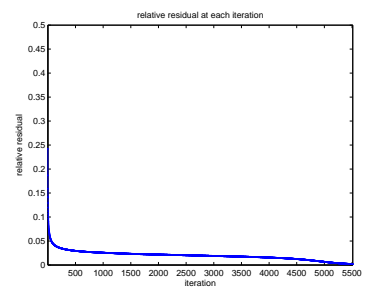

(j)

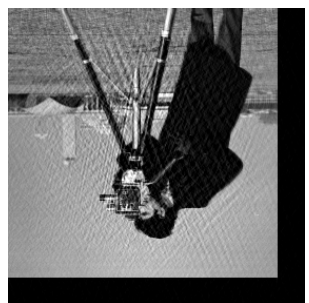

(c)

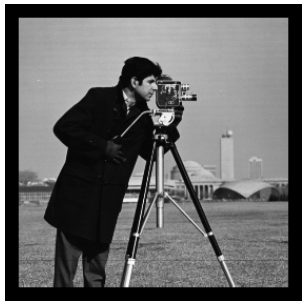

(g)

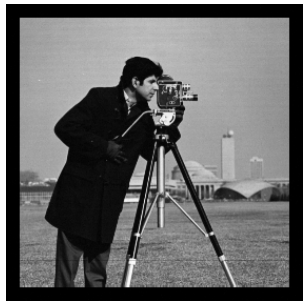

(k)

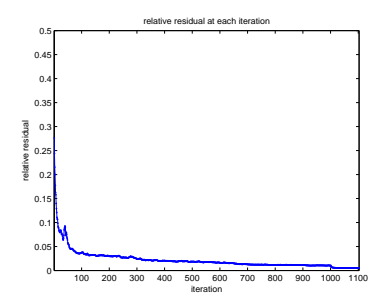

(d)

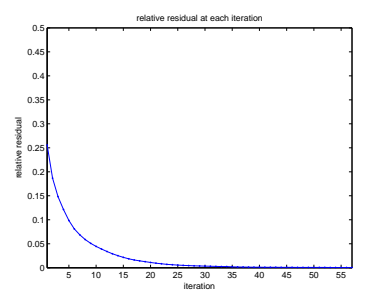

(h)

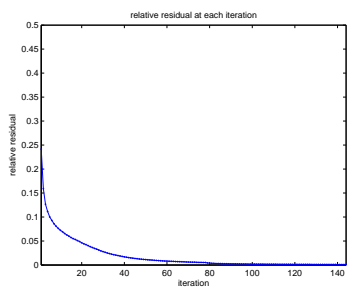

(1)

Fig. 3. (a) Recovery by 1651 ER iterations with UI and $\sigma=4$. (b) $r\left(f_{k}\right)$ versus $\mathrm{k}$ with $r(\hat{f}) \approx 5.49 \%$. (c) Recovery by $1000 \mathrm{HIO}+103 \mathrm{ER}$ with UI and $\sigma=4$. (d) $r\left(f_{k}\right)$ versus $\mathrm{k}$ with $r(\hat{f}) \approx 0.49 \%$. (e) Recovery by $1587 \mathrm{ER}$ steps with one low resolution RPI with $\sigma=2$. (f) $r\left(f_{k}\right)$ versus $\mathrm{k}$ with $r(\hat{f}) \approx 0.52 \%$ and $e(\hat{f}) \approx 2.51 \%$. (g) Recovery by $33 \mathrm{HIO}+24$ ER steps with low resolution RPI with $\sigma=2$. (h) $r\left(f_{k}\right)$ versus k with $r(\hat{f}) \approx 0.05 \%$ and $e(\hat{f}) \approx 0.32 \%$. (i) Recovery by 5512 ER steps with high resolution RPI with $\sigma=1$. (j) $r\left(f_{k}\right)$ versus $\mathrm{k}$ with $r(\hat{f}) \approx 0.19 \%$ and $e(\hat{f}) \approx 3.27 \%$. (k) Recovery by $77 \mathrm{HIO}+$ 67 ER steps with high resolution RPI with $\sigma=1$. (l) $r\left(f_{k}\right)$ versus $\mathrm{k}$ with $r(\hat{f}) \approx 0.10 \%$ and $e(\hat{f}) \approx 1.39 \%$. 


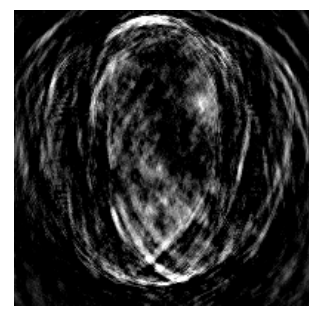

(a)

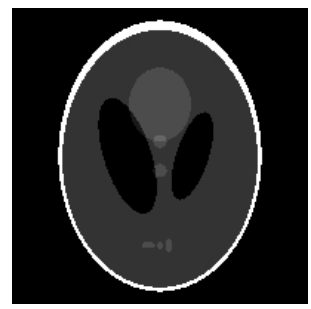

(e)

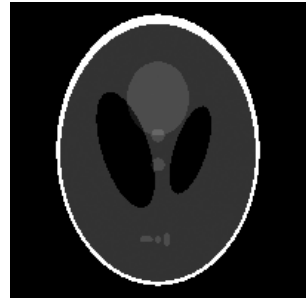

(i)

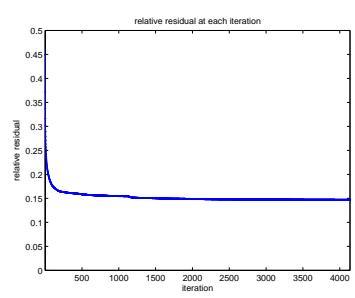

(b)

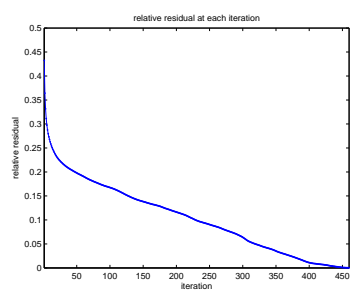

(f)

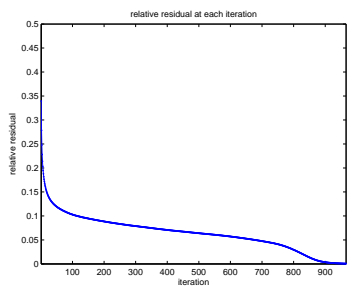

(j)

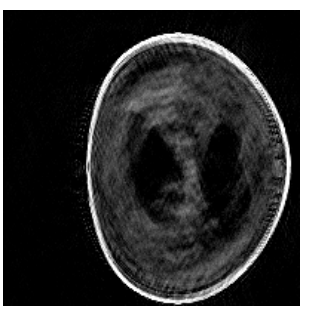

(c)

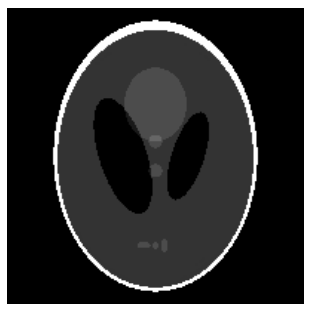

(g)

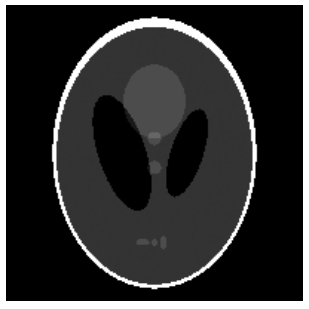

$(\mathrm{k})$

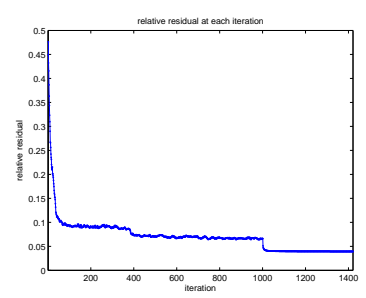

(d)

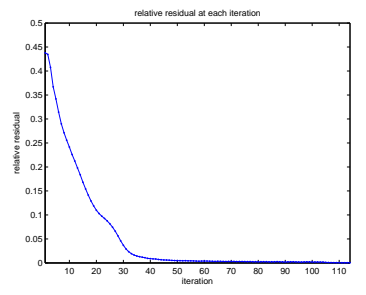

(h)

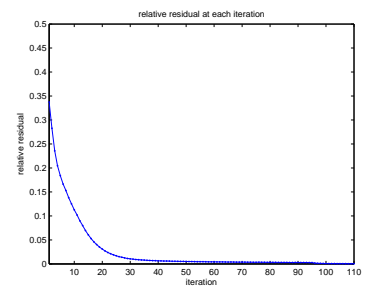

(l)

Fig. 4. (a) Recovery by 4140 ER iterations with UI with $\sigma=4$. (b) $r\left(f_{k}\right)$ versus $\mathrm{k}$ with $r(\hat{f}) \approx 14.71 \%$. (c) Recovery by 1000 HIO + 421 ER steps with one UI with $\sigma=4$. (d) $r\left(f_{k}\right)$ versus k with $r(\hat{f}) \approx 3.94 \%$. (e) Recovery by 460 ER with one low resolution RPI with $\sigma=2$. (f) $r\left(f_{k}\right)$ versus $\mathrm{k}$ with $r(\hat{f}) \approx 0.03 \%$ and $e(\hat{f}) \approx 0.09 \%$. (g) Recovery by $103 \mathrm{HIO}+11$ ER steps with one low resolution RPI with $\sigma=2$. (h) $r\left(f_{k}\right)$ versus $\mathrm{k}$ with $r(\hat{f}) \approx 0.03 \%$ and $e(\hat{f}) \approx 0.12 \%$. (i) Recovery by 966 ER steps with one high resolution RPI with $\sigma=1$. (j) $r\left(f_{k}\right)$ versus $\mathrm{k}$ with $r(\hat{f}) \approx 0.06 \%$ and $e(\hat{f}) \approx 0.40 \%$. (k) Recovery by $94 \mathrm{HIO}+$ 16 ER steps with one high resolution RPI with $\sigma=1$. (l) $r\left(f_{k}\right)$ versus $\mathrm{k}$ with $r(\hat{f}) \approx 0.04 \%$ and $e(\hat{f}) \approx 0.19 \%$. 


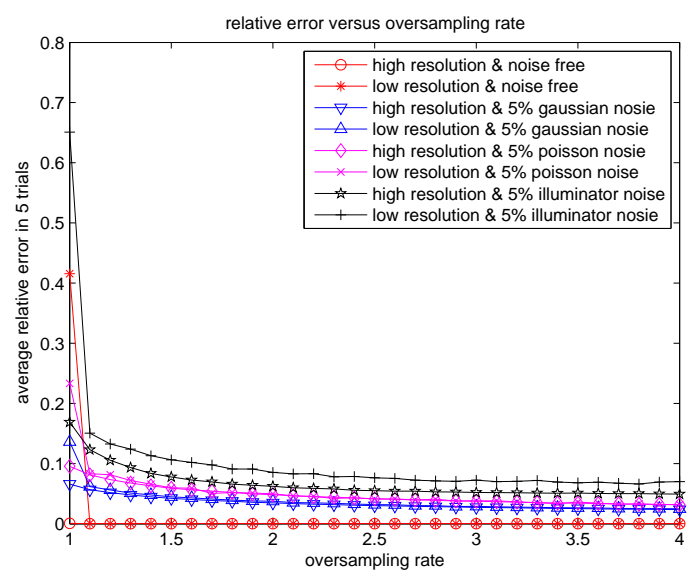

(a)

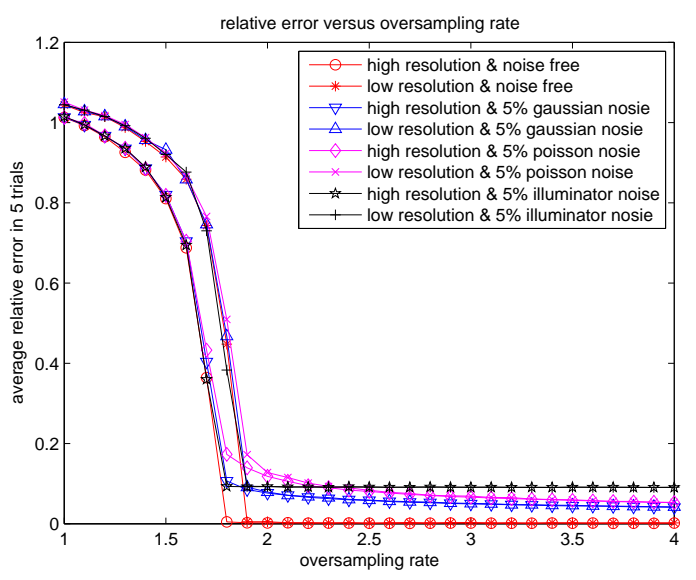

(b)

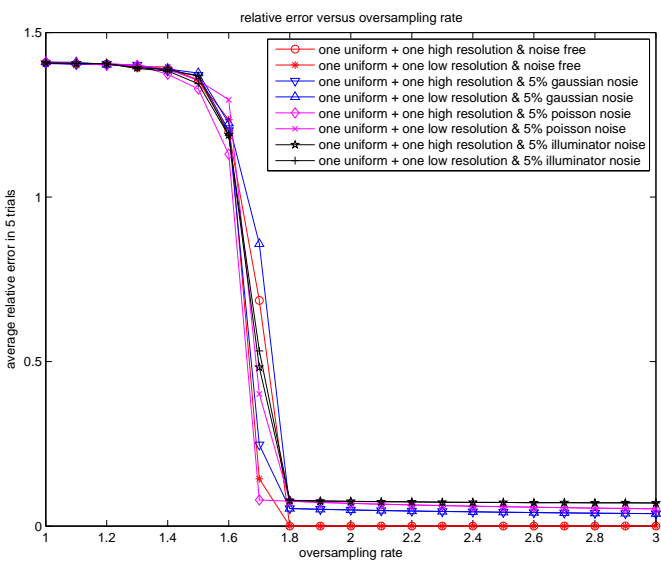

(c)

Fig. 5. (a) Relative error with one RPI for nonnegative-valued Phantom; (b) Relative error with one RPI for complex-valued Phantom with phases randomly distributed in $[0, \pi / 2]$; (c) Relative error by $200 \mathrm{HIO}+300 \mathrm{ER}$ with one RPI and UI for complex-valued Phantom with phases randomly distributed in $[0,2 \pi]$ with one UI and one RPI of high resolution (block size: $1 \times 1$ ) or low resolution (block size: $4 \times 4$ ). 


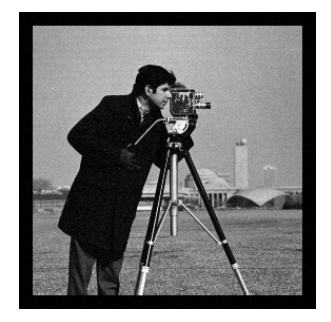

(a)

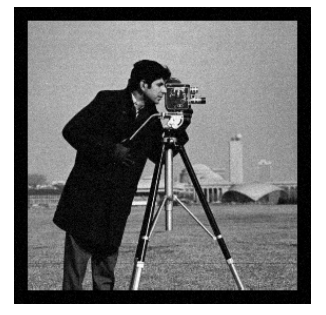

(e)

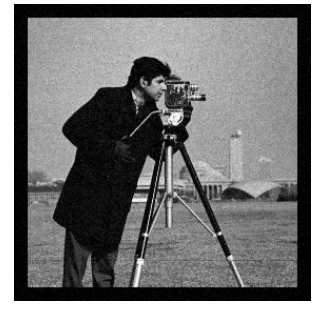

(i)

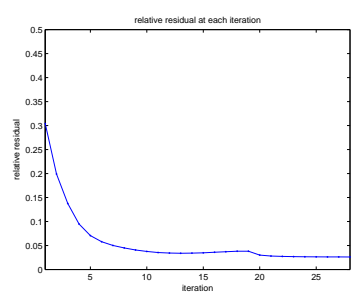

(b)

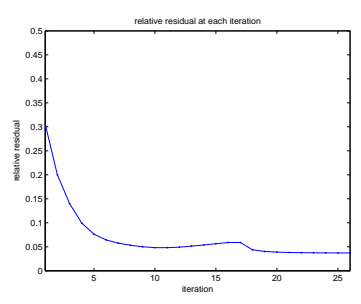

(f)

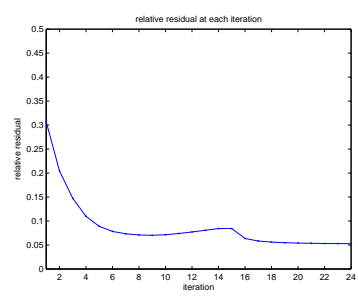

(j)

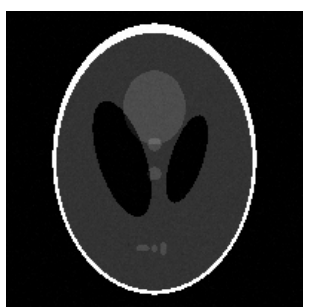

(c)

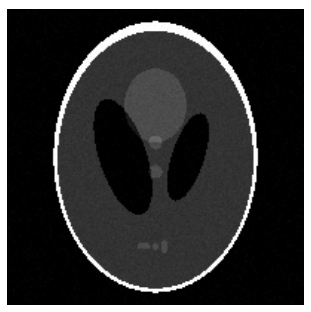

(g)

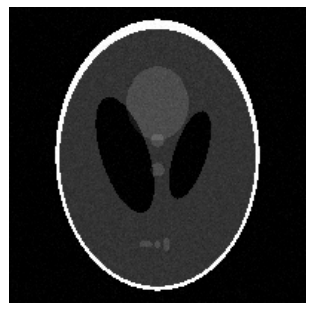

(k)

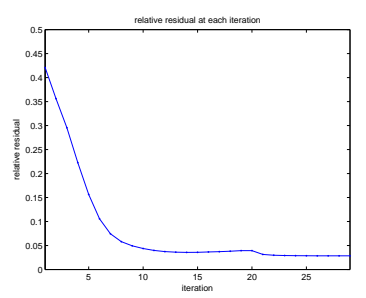

(d)

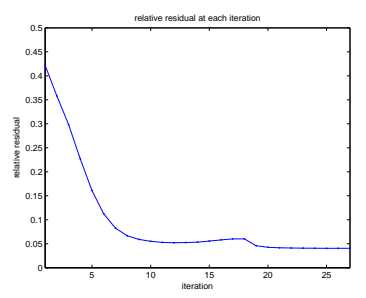

(h)

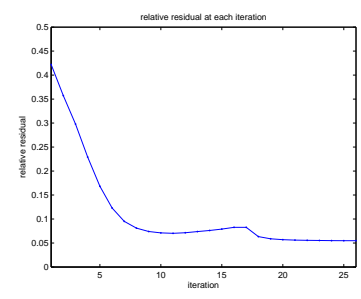

(l)

Fig. 6. Phasing with $\sigma=2$ and one high resolution RPI: (a) Recovery by 18 $\mathrm{HIO}+10$ ER with $5 \%$ Gaussian noise; (b) $r\left(f_{k}\right)$ versus $\mathrm{k}$ with $r(\hat{f}) \approx 2.62 \%$ and $e(\hat{f}) \approx 4.20 \%$; (c) Recovery by $19 \mathrm{HIO}+10$ ER with $5 \%$ Gaussian noise. (d) $r\left(f_{k}\right)$ versus k with $r(\hat{f}) \approx 2.85 \%$ and $e(\hat{f}) \approx 3.51 \%$; (e) Recovery by 16 $\mathrm{HIO}+10$ ER with $5 \%$ Poisson noise; (f) $r\left(f_{k}\right)$ versus k with $r(\hat{f}) \approx 3.71 \%$ and $e(\hat{f}) \approx 5.89 \%$; $(\mathrm{g})$ Recovery by $17 \mathrm{HIO}+10$ ER with $5 \%$ Poisson noise; $(\mathrm{h})$ $r\left(f_{k}\right)$ versus $\mathrm{k}$ with $r(\hat{f}) \approx 4.05 \%$ and $e(\hat{f}) \approx 4.84 \%$; (i) Recovery by $14 \mathrm{HIO}$ +10 ER with $5 \%$ illuminator noise; $(\mathrm{j}) r\left(f_{k}\right)$ versus $\mathrm{k}$ with $r(\hat{f}) \approx 5.28 \%$ and $e(\hat{f}) \approx 7.75 \%$; $(\mathrm{k})$ Recovery by $16 \mathrm{HIO}+10 \mathrm{ER}$ with $5 \%$ illuminator noise; (l) $r\left(f_{k}\right)$ versus $\mathrm{k}$ with $r(\hat{f}) \approx 5.48 \%$ and $e(\hat{f}) \approx 6.35 \%$. 


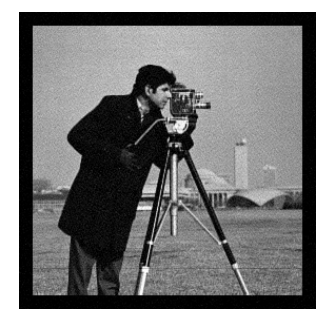

(a)

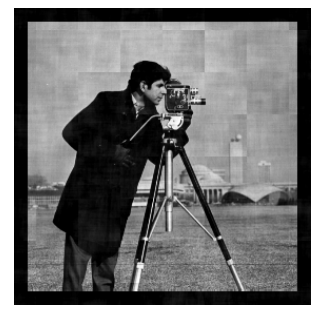

(e)

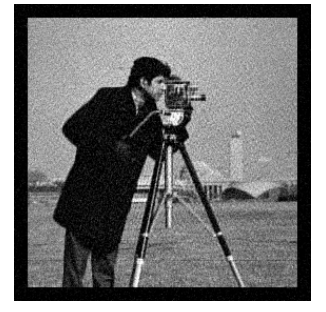

(i)

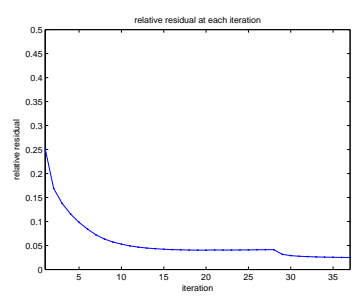

(b)

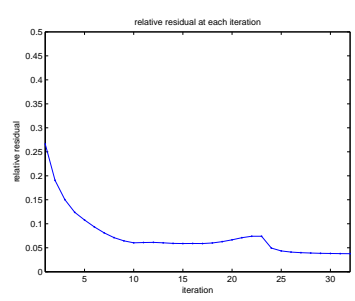

(f)

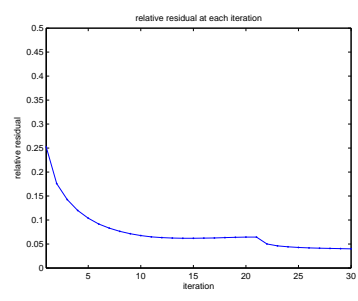

(j)

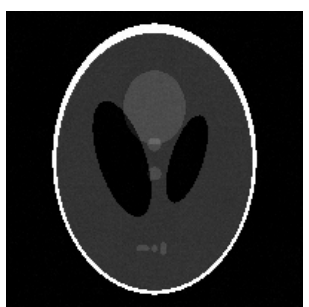

(c)

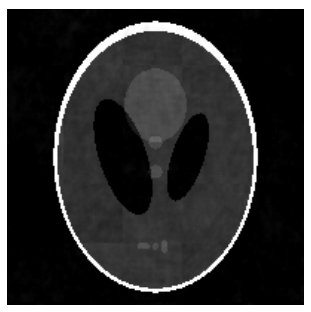

(g)

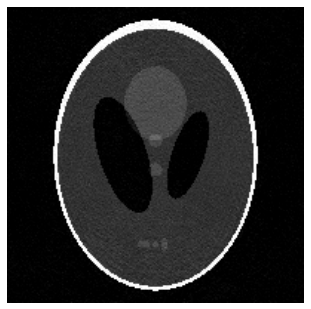

(k)

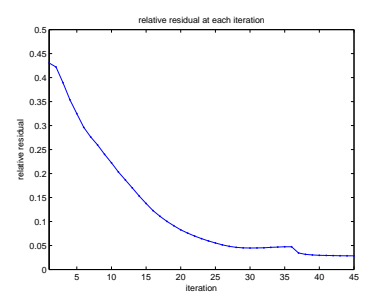

(d)

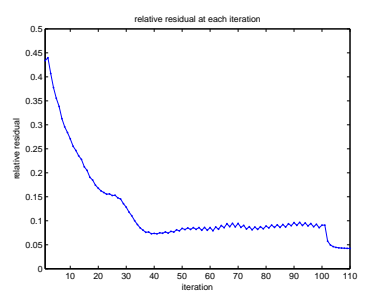

(h)

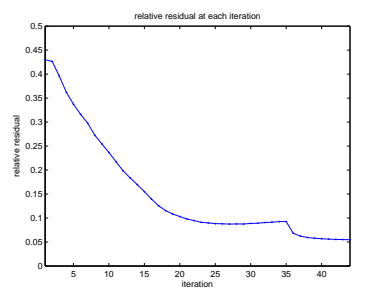

(l)

Fig. 7. Phasing with $\sigma=2$ and one low $(40 \times 40)$ resolution RPI: (a) Recovery by $27 \mathrm{HIO}+10 \mathrm{ER}$ with $5 \%$ Gaussian noise; (b) $r\left(f_{k}\right)$ versus k with $r(\hat{f}) \approx$ $2.50 \%$ and $e(\hat{f}) \approx 7.37 \%$; (c) Recovery by $35 \mathrm{HIO}+10 \mathrm{ER}$ with $5 \%$ Gaussian noise. (d) $r\left(f_{k}\right)$ versus k with $r(\hat{f}) \approx 2.85 \%$ and $e(\hat{f}) \approx 4.18 \%$; (e) Recovery by $22 \mathrm{HIO}+10 \mathrm{ER}$ with $5 \%$ Poisson noise; (f) $r\left(f_{k}\right)$ versus $\mathrm{k}$ with $r(\hat{f}) \approx 3.77 \%$ and $e(\hat{f}) \approx 6.27 \%$; $(\mathrm{g})$ Recovery by $100 \mathrm{HIO}+10$ ER with $5 \%$ Poisson noise; (h) $r\left(f_{k}\right)$ versus $\mathrm{k}$ with $r(\hat{f}) \approx 4.24 \%$ and $e(\hat{f}) \approx 5.09 \%$; (i) Recovery by 20 $\mathrm{HIO}+10$ ER with $5 \%$ illuminator noise; $(\mathrm{j}) r\left(f_{k}\right)$ versus $\mathrm{k}$ with $r(\hat{f}) \approx 4.00 \%$ and $e(\hat{f}) \approx 13.14 \%$; $(\mathrm{k})$ Recovery by $34 \mathrm{HIO}+10 \mathrm{ER}$ with $5 \%$ illuminator noise; (l) $r\left(f_{k}\right)$ versus $\mathrm{k}$ with $r(\hat{f}) \approx 5.48 \%$ and $e(\hat{f}) \approx 9.46 \%$. 


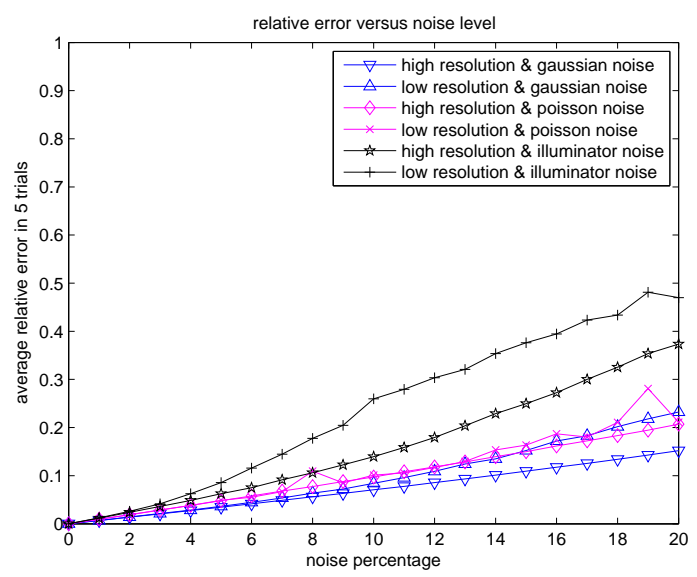

(a)

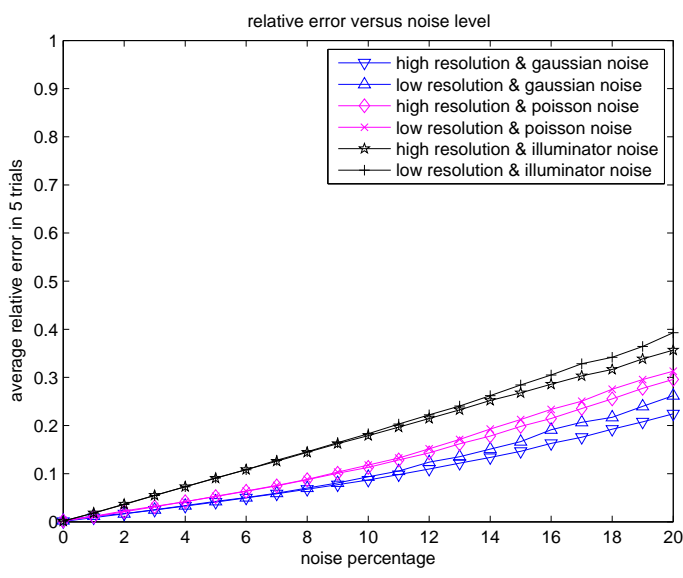

(b)

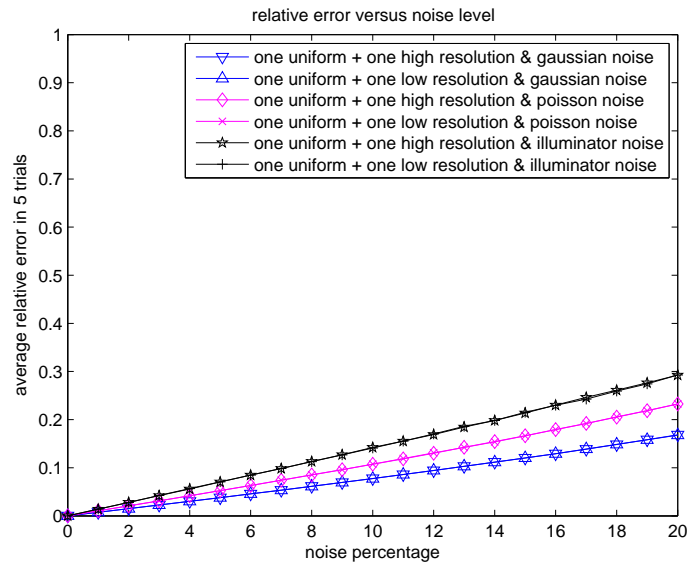

(c)

Fig. 8. (a) Relative error for nonnegative-valued Phantom and $\sigma=2$ (b) Relative error for complex-valued Phantom with phases randomly distributed in $[0, \pi / 2]$ and $\sigma=4$; (c) Relative error for complex-valued Phantom with phases randomly distributed in $[0,2 \pi]$ and $\sigma=3$. 